\title{
ROMANIAN
}

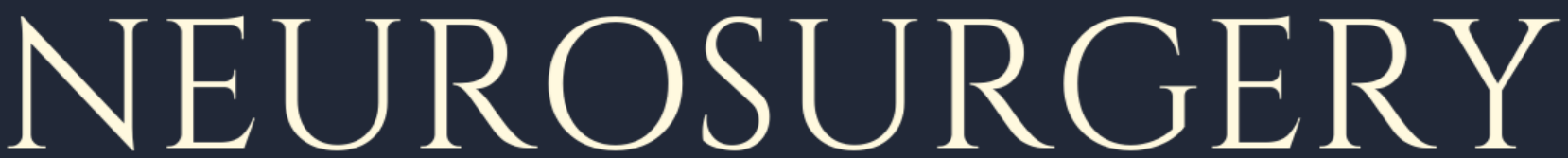

\author{
Vol. XXXIV | No. $1 \quad$ March 2020
}

\section{Lumbar unilateral laminotomy for bilateral decompression in lumbar spinal stenosis}

\author{
Amin Sabry, \\ Wael Zakarya, \\ Amr Farid Khalil
}




\title{
Lumbar unilateral laminotomy for bilateral decompression in lumbar spinal stenosis
}

\author{
Amin Sabry, Wael Zakarya, Amr Farid Khalil
}

Neurosurgery Department, Mansoura University, EGYPT

\begin{abstract}
Background: Multiple surgical approaches are existing for the management of lumbar canal stenosis.

Objective: This study was conducted to assess the outcomes of unilateral laminotomy with bilateral decompression in such cases.

Patients and methods: This prospective study was conducted at Mansoura University Hospitals, and we included a total of 12 cases with lumbar canal stenosis. All cases underwent unilateral laminotomy with bilateral canal decompression during the period between July 2017 and July 2018. Post-operative outcomes included ODI, and VAS score for both leg and back pain.

Results: The age of the cases ranged between 38 and 62 years. We included 7 males and 5 females. ODI, lower extremity, and back pain showed a significant decrease after the operation $(p<0.05)$.

Conclusion: Unilateral laminotomy with bilateral canal decompression is a safe and feasible approach to managing LSS. Excellent outcomes are expected regarding leg pain and quality of life, while slight improvement is anticipated regarding low back pain.
\end{abstract}

\section{INTRODUCTION}

Lumbar spine stenosis (LSS) is one of the commonest spinal pathologies, that present with buttock or lower limb pain associated with decreased neurovascular space in the lumbar spine region. Low back pain may be present or not [2].

Degenerative LSS usually starts in the 5th or 6th decades of life. It is characterized by hypertrophy of ligamentum flavum, intervertebral disc bulging, thickening of the facet joint, and arthropathy. These changes lead to inevitable canal narrowing $[2,15]$. Cases may express intermittent neurological claudications, and the quality of life is markedly decreased [13].

Although conservative treatment can provide a temporary symptom relief, surgical decompression will be eventually needed. Nowadays, multiple surgical approaches are existing for management of such disorder. There is no definite data favoring one technique over another one [8].

Conventional laminectomy is the commonest approach performed for degenerative LSS [5]. However, the integrity of posterior spine

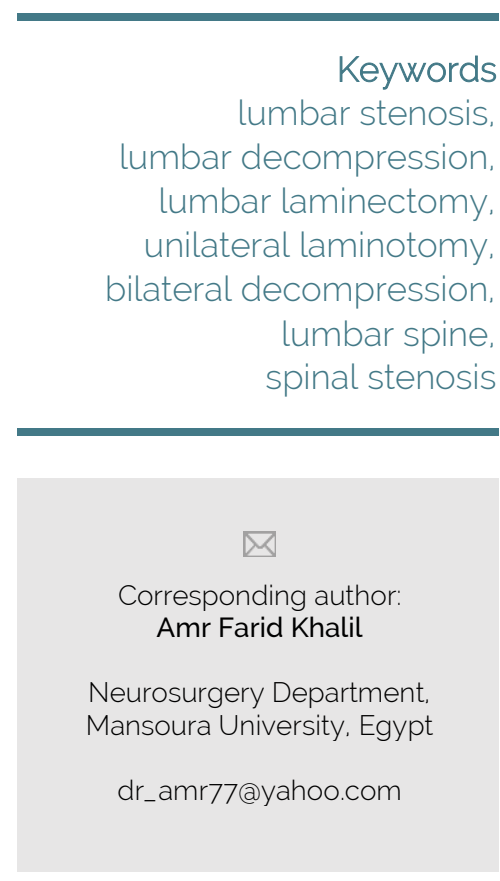

Copyright and usage. This is an Open Access article distributed under the terms of the Creative Commons Attribution Non-Commercial No Derivatives License (https://creativecommons org/licenses/by-nc-nd/4.0/) which permits noncommercial re-use, distribution, and reproduction in any medium, provided the original work is unaltered and is properly cited.

The written permission of the Romanian Society of Neurosurgery must be obtained for commercial re-use or in order to create a derivative work.

ISSN online 2344-4959 (C) Romanian Society of Neurosurgery

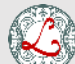

First published

London Academic Publishing www.lapub.co.uk 
complex is negatively affected. Furthermore, elevation of paravertebral muscles from the spinous process leads to spinal muscle atrophy and weakness in the trunk extensors [2].

As most of LSS patients are of old age, multiple comorbidities are usually present. Thus, the invasiveness of surgery must be kept into consideration since more invasive procedures are associated with higher morbidity and mortality rates along with increased health costs [13].

Unilateral laminotomy with bilateral decompression provides the advantage of preserving neural arch and facet joint of the other side. Hence, stability is more preserved, and neural tissue are protected against posterior scarring. Besides, it offers shorter operative time, less blood loss, and less post-operative morbidity when compared to the conventional approach $[3,10,12]$.

The success rate of unilateral approach in patients with bilateral canal decompression ranges between 68 and 94\% [2].

This study was conducted at Mansoura University Hospitals aiming to evaluate the outcome of unilateral laminotomy with bilateral canal dilatation in LSS.

\section{PATIENT AND METHODS}

\section{Study design}

This is a prospective study that was conducted during the period between July 2017 and July 2018.

Study cases

A total of 12 cases with degenerative LSS were included in the study. All cases experienced failure of medical treatment or physiotherapy for at least 3 months. Cases with neurological claudication or radiculopathy, and radiological features of LSS were included. Cases with previous spine surgery, spinal tumours, instability, or spondylolisthesis were excluded.

Patient consent

A pre-operative written informed consent was obtained from all cases after the explanation of advantages and drawbacks of the surgical approach. Moreover, the study was approved by the local ethical committee.

Patient preparation

All cases were subjected to complete history taking, thorough physical examination, and routine laboratory investigations. Besides, an MRI of the lumbosacral spine was ordered for all cases. In addition, plain $\mathrm{X}$ ray was also performed to exclude instability.

Pain was assessed via visual analogue score (VAS), while functional status was evaluated by Oswestry Disability Index (ODI).

\section{Surgical procedure}

The operation was performed when the patient was in prone position. A midline incision was created over the stenotic area as localized in pre-operative MRI. By the aid of microscope or vascular loupe, a unilateral laminotomy was carried out, whereas the inferior aspect of cranial hemilamina and the superior aspect of the caudal hemilamina were partially resected. The spinous process base was undercut after ipsilateral decompression. Then, bilateral flavectomy with contralateral neural foramen decompression was done. Following bilateral decompression, the nerve roots were visualized easily and th that point, the operation was ended.

\section{Post-operative care and follow up}

All cases were transferred to the recovery room, them to the internal ward. Mobilization was encouraged on the 1st post-operative day. Postoperative VAS and ODI were recorded at 6-and 12month visits. Post-operative radiological evaluation of stability was not routinely performed unless the patient is still complaining of back pain or claudication is still existing.

\section{Statistical analysis}

The collected data were coded, processed and analyzed using the SPSS (Statistical Package for Social Sciences) version 22 for Windows ${ }^{\circledR}$ (IBM, SPSS Inc, Chicago, IL, USA).

Data were tested for normal distribution using the Shapiro Walk test. Qualitative data were represented as frequencies and relative percentages. Quantitative data were expressed as median (Range). Kruskal Wallis test (KW) was used to test the significance between values at more than two time points (preoperative, at 6 months and at 12 months). Wilcoxon signed rank test was used to test significance between two different time points. For all tests, $p$ value $(<0.05)$ was considered significant. 


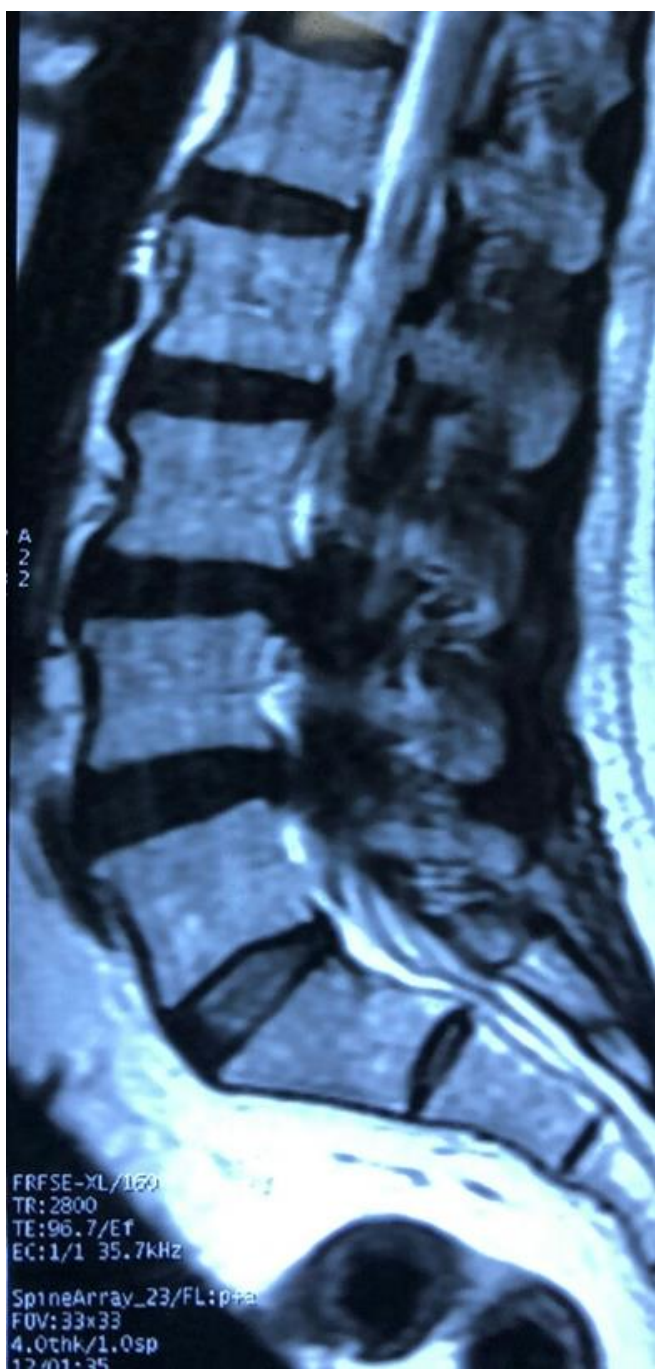

Figure 1. Sagittal T2 pre-operative MRI shows severe spinal canal stenosis with multiple disc bulge and ligamentum flavum thickening.

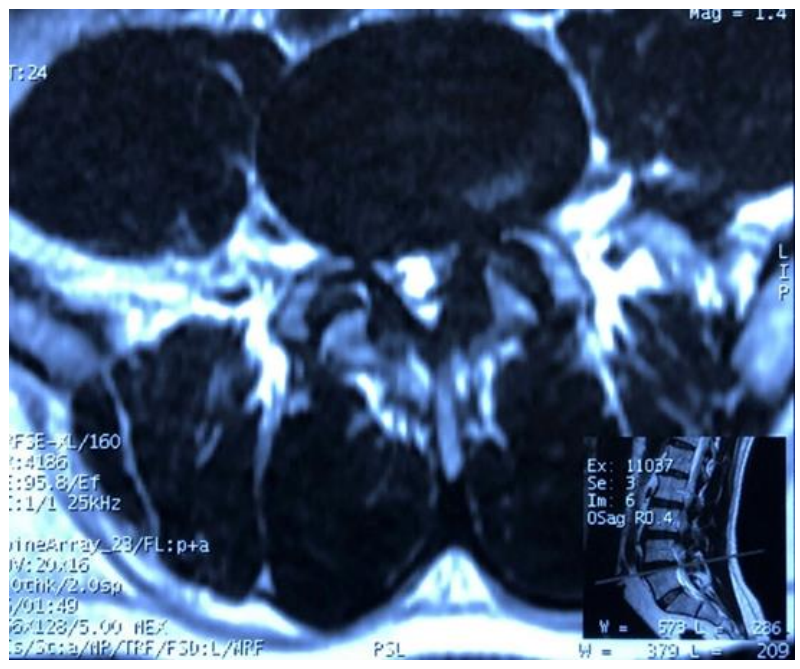

Figure 2. Axial T2 pre-operative MRI shows Severe spinal canal stenosis with a disc bulge and ligamentum flavum thickening.

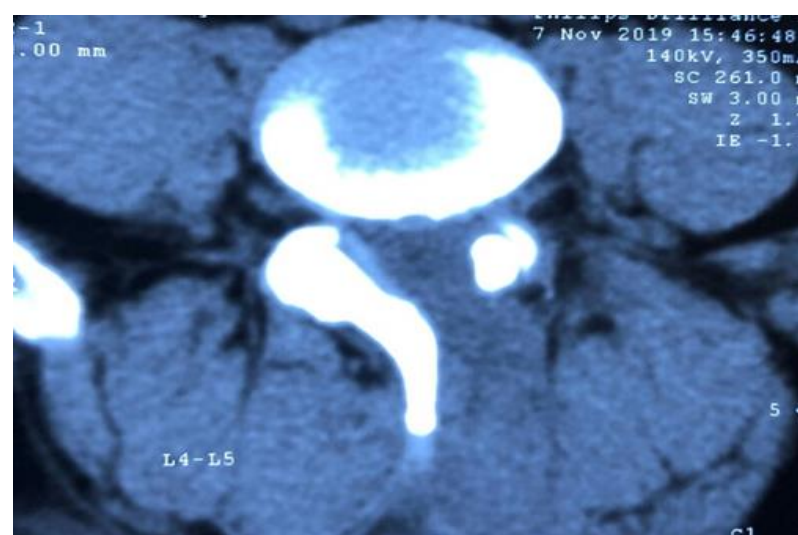

Figure 3. Axial CT scans L4 spine after the patient underwent a left L4 Unilateral laminotomy.

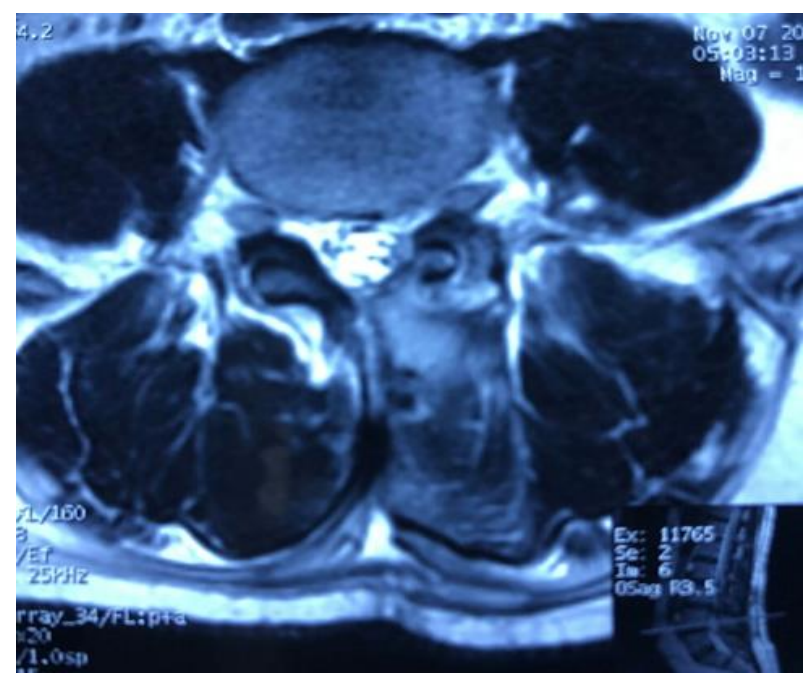

Figure 4. Axial T2 Post-operative MRI shows marked widening of the spinal canal after left Unilateral laminotomy.

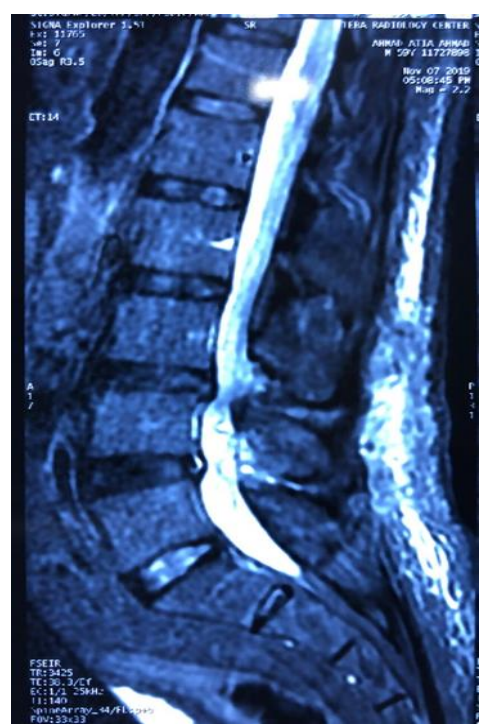

Figure 5. Sagittal T2 post-operative MRI after double level (L3L4 and L4-L5 decompression) via Unilateral laminotomy. 


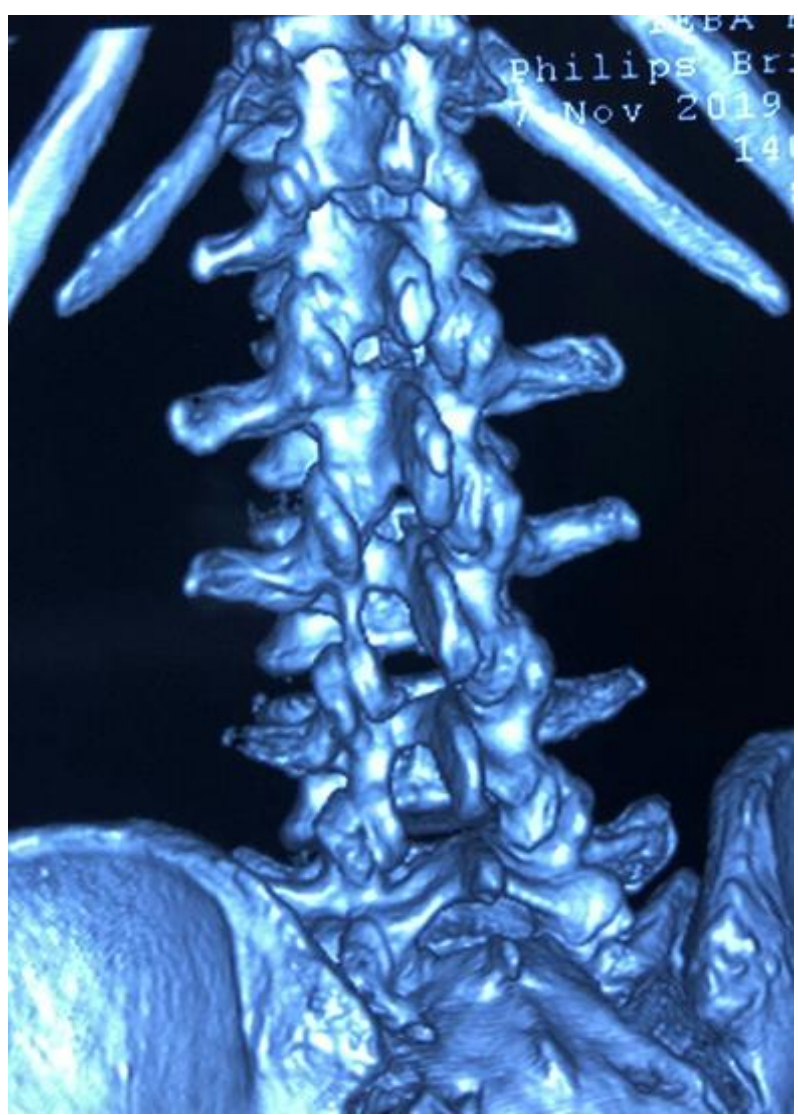

Figure 6. Post-operative CT 3D lumber spine after double level (L3-L4 and L4-5 Unilateral laminotomy).

\section{RESULTS}

The age of the included cases ranged between 38 and 62 years (median $=56$ ). We included 7 males (58.33\%) and 5 females (41.66\%). These data are shown at table (1).

Preoperative ODI ranged between 22 and 36 (median = 28). It decreased significantly after operation down to 7 and 6 scores at 6-month and 1years follow up visits $(p<0.001)$.

After operation VAS score decreased down to 1 at the scheduled follow up visits. It has a median value of 9 before operation. Table (3) illustrates these data. Back pain also significantly decreased after operation from score 6 preoperatively down to 3 and 2 scores after 6 and 12 months respectively. These data are illustrated at table (4).

Table 1. Patient criteria.

\begin{tabular}{|l|l|}
\hline Variable & Data \\
\hline Age & $56(38-62)$ \\
\hline Sex & \\
-Male & $7(58.33 \%)$ \\
-Female & $5(41.66 \%)$ \\
\hline
\end{tabular}

Table 2. ODI before and after surgery.

\begin{tabular}{|l|l|l|l|}
\hline & Preoperatively & After 6 months & After 1 year \\
\hline & $28(22-36)$ & $7(3-11)$ & $6(3-10)$ \\
\hline P1 & & $<0.001^{*}$ & $<0.001^{*}$ \\
\hline P2 & & & 0.145 \\
\hline P & \multicolumn{3}{|c|}{$<0.001^{*}$} \\
\hline
\end{tabular}

$\mathrm{P}$ : significance between different durations P1: significance in relation to preoperative value. P2: significance in relation to 6 months value. *: statistically significant $(p<0.05)$

Table 3. VAS score for limb pain before and after surgery.

\begin{tabular}{|l|l|l|l|}
\hline & Preoperatively & $\begin{array}{l}\text { After } \\
\text { months }\end{array}$ & After 1 year \\
\hline & $9(7-10)$ & $1(1-3)$ & $1(0-2)$ \\
\hline P1 & & $0.001^{*}$ & $<0.001^{*}$ \\
\hline P2 & \multicolumn{3}{|c|}{$<0.001^{*}$} \\
\hline P & \multicolumn{3}{|c|}{0.124} \\
\hline
\end{tabular}

$\mathrm{P}$ : significance between different durations P1: significance in relation to preoperative value. P2: significance in relation to 6 months value. *: statistically significant $(p<0.05)$

Table 4. VAS score for back pain before and after surgery.

\begin{tabular}{|l|l|l|l|}
\hline & Preoperatively & After 6 months & After 1 year \\
\hline & $6(4-7)$ & $3(2-4)$ & $2(1-4)$ \\
\hline P1 & & $0.009^{*}$ & $0.001^{*}$ \\
\hline P2 & \multicolumn{3}{|c|}{$0.005^{*}$} \\
\hline P & \multicolumn{3}{|c|}{0.108} \\
\hline
\end{tabular}

P: significance between different durations $\mathrm{P} 1$ : significance in relation to preoperative value. P2: significance in relation to 6 months value. *: statistically significant $(p<0.05)$

\section{DISCUSSION}

Multiple surgical approaches have been proposed for the surgical management of LSS. The main surgical goal is to decompress the lumbar canal. However, anatomy should be preserved to maintain the biomechanical function of the lumbar spine [2].

On using the conventional decompression technique, some authors reported paraspinal muscle atrophy on CT scan [7], and others showed electromyographic abnormalities following that approach [14]. These changes can lead to spinal instability and increase the need for spinal fusion surgery [6].

Conversely, the unilateral approach decrease the incidence of these complications after surgery [13]. 
This study was conducted at Mansoura University Hospitals aiming to evaluate the surgical outcomes of unilateral laminotomy with bilateral decompression for LSS.

A total of 12 cases were included with a median age of 56 (range, 38-62). We included 7 males (58.33\%) and 5 females (41.66\%).

Another Egyptian study handled the same perspective included 21 cases in the unilateral approach group, with a mean age of 47.2 years (range, 33 - 69). The presence of young age groups is due to the presence of cases with disco-ligamentous causes of LSS, which is more common in young age due to sedentary life and overweight (like in Egyptian population), compared to the bony type which is common in the older population [1].

In the current study, the operation successfully decreased ODI score from 28 preoperatively down to 7 and 6 scores 6 and 12 months after operation ( $p$ $<0.001$ ).

Regarding ODI in other studies, it decreased from 28.7 preoperatively down to 5.55 and 6.5 at 1 -month and 1-year follow-up visits respectively. However, that change was not significantly different from the conventional approach group [1].

Another study used both Japanese Orthopedic Association Score (JOA) and Neurogenic Claudication Outcome Score (NCOS) to evaluate the outcomes in unilateral laminectomy patients. There was a significant increase in both parameters form 4.35 and 26.9 up to 10.2 and 61.15 after operation respectively. This improvement was also better than the conventional approach group. That study reported that excellent and good outcomes were achieved in 14 cases in the unilateral hemilaminectomy group (70\%) [13].

On assessment of leg pain in the current study, it decreased from 9 preoperatively (range, $7-10$ ), down to 1 at the scheduled follow up visits $(p<$ $0.001)$.

In the previously mentioned Egyptian study, the pre-operative VAS score for lower extremity pain was 9.04 , and it decreased significantly down to 1.38 and 1.46 at 1 -month and 12-month follow-up visits ( $p=$ 0.001) [1].

This comes in line with the findings of Çavuşoğlu and his colleagues who stated that most VAS changes occur between operation and early follow up [4].
When it comes to back pain in our study, it was also assessed via VAS score which decreased significantly from 6 (range, $4-7$ ) before operation, to 3 (range, $2-4)$ after 6 months $(p=0.009)$, and 2 (range, $1-4)$ after 1 year $(p=0.001)$.

In another study, the mean value of preoperative VAS score for back pain was 5.42. It decreased slightly down to 2.82 and 1.96 at 1-month and 1-year follow-up visits respectively [1].

Another study has also published that VAS score for back pain has decreased from 7.6 pre-operatively down to 2.95 after operation. This decrease was also more significant when compared to the conventional approach [13].

Another recent study also stated that detailed lower back pain VAS score before surgery was 51.5 in motion, 63.0 while standing, and 37.8 while sitting; and showed LBP while standing was significantly greater than LBP while sitting $(p<0.01)$. After surgery, LBP while standing was significantly improved relative to that while sitting $(p<0.05)$, and levels of LBP in the three postures became almost the same with ODI improvement. Bilateral VAS scores showed significant improvement equally on both sides $(p<0.01)[16]$.

Regarding complications encountered in the current study, durotomy was encountered in only one case (8.33\%), who was managed conservatively.

Other authors reported that unintended durotomy occurred in $4.5 \%$ of their cases [11]. Another study reported that that complication occurred in about $5-15 \%$ of cases [4]. In addition, $\mathrm{Ng}$ and his colleagues also reported that the incidence of that complication was 14\% [9]. This comes in line with our results.

In the study conducted by Abbas and his associates, early post-operative complications were encountered in 2 cases (18.2\%); one had CSF leak, and the other had a wound hematoma [1].

The main disadvantage with our study is the relatively small sample size. So, more studies with larger sample size should be conducted in the near future.

\section{CONCLUSION}

Unilateral laminotomy with bilateral canal decompression is a safe and feasible approach in managing LSS. Excellent outcomes are expected regarding leg pain and quality of life, while slight improvement is anticipated regarding low back pain. 


\section{REFERENCES}

1. Abbas M, Elwany A, and Farag R: Efficacy and Safety of Using Unilateral Approach for Bilateral Neural Decompression in Lumbar Spinal Stenosis. Egyptian Spine Journal 2017, 24(1),42-9.

2. Amir S, Qadir M, Pasha I F, and Qureshi M A: To determine the efficacy of unilateral approach for Bilateral Decompression in Lumbar Spinal Stenosis. Journal of Saidu Medical College 2018, 7(2).

3. AYDIN Y: Efficacy of unilateral laminectomy for bilateral decompression in lumbar spinal stenosis. Turkish neurosurgery 2007, 17(2),100-8.

4. Çavuşoğlu H, Kaya R A, Türkmenoglu O N, Tuncer C, Colak I, and Aydın Y: Midterm outcome after unilateral approach for bilateral decompression of lumbar spinal stenosis: 5-year prospective study. European Spine Journal 2007, 16(12),2133-42.

5. Galiano K, Obwegeser A A, Gabl M V, Bauer R, and Twerdy $\mathrm{K}$ : Long-term outcome of laminectomy for spinal stenosis in octogenarians. Spine 2005, 30(3),332-5.

6. Lipson S J: Spinal-fusion surgery-advances and concerns. New England Journal of Medicine 2004, 350(7),643-4.

7. Mayer T G, Vanharanta H, Gatchel R J, Mooney V, Barnes $D$, Judge L, Smith S, and Terry A: Comparison of CT scan muscle measurements and isokinetic trunk strength in postoperative patients. Spine 1989, 14(1),33-6.

8. Moisi M, Fisahn C, Tkachenko L, Tubbs R S, Ginat $D$, Grunert P, Jeyamohan S, Reintjes S, Ajayi O, and Page J: Unilateral laminotomy with bilateral spinal canal decompression for lumbar stenosis: a technical note. Cureus 2016, 8(5).

9. Ng L C, Tafazal S, and Sell P: The effect of duration of symptoms on standard outcome measures in the surgical treatment of spinal stenosis. European Spine Journal 2007, 16(2),199-206.
10. Oertel M F, Ryang Y-M, Korinth M C, Gilsbach J M, and Rohde $\mathrm{V}$ : Long-term results of microsurgical treatment of lumbar spinal stenosis by unilateral laminotomy for bilateral decompression. Neurosurgery 2006, 59(6),126470.

11. Podichetty V K, Spears J, Isaacs R E, Booher J, and Biscup R S: Complications associated with minimally invasive decompression for lumbar spinal stenosis. Clinical Spine Surgery 2006, 19(3),161-6.

12. Rahman M, Summers L, Richter B, Mimran R, and Jacob $\mathrm{R}$ : Comparison of techniques for decompressive lumbar laminectomy: the minimally invasive versus the "classic" open approach. min-Minimally Invasive Neurosurgery 2008, 51(02),100-5.

13. Ramesh K, and Vimalan G: Comparative study between functional outcome of lumbar canal stenosis treated with surgical decompression by laminectomy and unilateral partial hemi laminectomy approaches. International Journal of Research in Orthopaedics 2018, 4(4),610.

14. See D, and Kraft G: Electromyography in paraspinal muscles following surgery for root compression. Archives of physical medicine and rehabilitation 1975, 56(2),80-3.

15. Steurer J, Nydegger A, Held U, Brunner F, Hodler J, Porchet F, Min K, Mannion A F, Michel B, and Collaboration L R: LumbSten: the lumbar spinal stenosis outcome study. BMC musculoskeletal disorders 2010, 11(1),254.

16. Takahashi $H$, Aoki $Y$, Saito J, Nakajima A, Sonobe $M$, Akatsu Y, Inoue M, Taniguchi S, Yamada M, and Koyama $\mathrm{K}$ : Unilateral laminectomy for bilateral decompression improves low back pain while standing equally on both sides in patients with lumbar canal stenosis: analysis using a detailed visual analogue scale. BMC musculoskeletal disorders 2019, 20(1),100. 